\title{
Studies of buried interfaces by optical second-harmonic generation
}

\author{
T. Rasing \\ Research Institute for Materials, Toernooiveld, NL-6525 ED Nijmegen, The Netherlands
}

Received 1 July 1994/Accepted 30 July 1994

\begin{abstract}
Optical Second-Harmonic Generation (SHG) has been used to study the technologically very important buried semiconductor-metal and magnetic multilayer interfaces. For the case of GaAs-Au, the SHG intensity is shown to depend on the applied bias and Schottky barrier height, and is strongly affected by the sweepout of the carriers generated by the fs excitation pulses. For the $\mathrm{M} / \mathrm{Co} / \mathrm{M}$ multilayers, with $\mathrm{M}=\mathrm{Cu}$ or $\mathrm{Au}$, the SHG signals appear to depend strongly on the magnetization and can be shown to be interface specific.
\end{abstract}

PACS: $42.65,73.30,75.70$

Nonlinear optical techniques like Second-Harmonic Generation (SHG) and Sum-Frequency Generation (SFG) have been proven to be versatile and sensitive surface and interface probes, that derive their surface specificity from the symmetry breaking at interfaces $[1,2]$. In particular, they are unique for studying buried interfaces, that are practically inaccessible by other than optical techniques. Such interfaces, like semiconductormetal, metal-metal, metal-electrolyte and various other interfaces, are highly relevant from a practical point of view, but also pose many fundamental questions about the interfacial electronic structure and dynamics. In this paper two of such interface problems will be adressed: the electric-field dynamics at a Schottky barrier and the magnetic structure of interfaces between thin films of magnetic and nonmagnetic materials. Though both studies rely on field-induced effects (electrical and magnetic, respectively), the observed signals appear to be quite large and to contain some interesting new results.

Paper presented at the 129th WE-Heraeus-Seminar on "Surface Studies by Nonlinear Laser Spectroscopies", Kassel, Germany, May 30 to June 1, 1994

\section{SHG at a metal-semiconductor interface}

The problem of Schottky barrier formation at a metalsemiconductor interface still attracts a lot of attention, as new developments in material fabrication and structure determination have shown that the precise interface structure plays a crucial role in determining the Schottky barrier height. For electro-optic applications, not only the static electronic structure but even more importantly, the dynamics near these interfaces in extremely relevant, as this will affect the speed of such devices. Recent photoluminescence studies of a $\mathrm{Au} / \mathrm{GaAs}$ structure have shown unexpected field and laser intensity dependences at quite moderate laser powers $(10 \mathrm{~mW})$ that are commonly met in optical semiconducting devices $[3,4]$. Theoretical models suggest a very rapid drop of the effective barrier height as a result of the spatial separation of the carriers excited by the incident laser puls (carrier sweepout). However, the photoluminescence is only indirectly affected by this interface field, as the signal is coming from a large bulk region. Very recently, Qi et al. [5] showed the power of SHG to probe the band bending region in GaAs. A direct way to probe the (time evolution of the) effective Schottky barrier height would be to measure the field induced SHG with ultra-short excitation pulses.

For an incident electric field $\mathrm{E}(\omega)$ the reflected SHG field follows from the nonlinear polarization $\mathbf{P}(2 \omega)$. In the electric dipole approximation $\mathbf{P}(2 \omega)$ is related to $\mathbf{E}(\omega)$ and an applied dc electric field $\mathrm{E}_{\mathrm{SB}}(0)$ via nonlinear susceptibility tensors as

$$
\begin{aligned}
\mathbf{P}(2 \omega)= & \left(\chi_{s}^{(2)}+\chi_{B}^{(2)}\right): \mathbf{E}(\omega) \mathbf{E}(\omega) \\
& +\left(\chi_{s}^{(3)}+\chi_{B}^{(3)}\right) \vdots \mathbf{E}(\omega) \mathbf{E}(\omega) \mathbf{E}_{S \mathrm{~B}}(0),
\end{aligned}
$$

where $\chi_{S}$ and $\chi_{B}$ are the interface and bulk nonlinear susceptibility tensors, respectively. Since GaAs has no inversion symmetry there are both bulk and interface nonzero tensor elements. The GaAs $(100)$ surface has $4 \mathrm{~mm}$ symmetry, and the three independent nonzero in- 
terface susceptibility elements are $\chi_{s ; z z z}^{(2)}, \chi_{s ; z i i}^{(2)}$ and $\chi_{s ; i z i}^{(2)}$ with $i=x, y[1,6]$. For the bulk of the $43 \mathrm{~m}$ symmetric $\mathrm{GaAs}(100)$ crystal there is only one independent nonzero susceptibility element $\chi_{B}^{(2)}=\chi_{B ; i j k}^{(2)}$ with $i \neq j \neq k$ [6]. The subscripts $(i, j, k)$ refer to the principal axes $(x, y, z)$ of the cubic crystal, with $z$ along the interface normal. The second term in (1) describes the so called electric field induced second-harmonic generation.

This field $\mathrm{E}_{S B}(0)$ has only a $z$-component, which we will indicate with $E_{\mathrm{SB}}$. The fourth rank tensors in the second term of (1) reduce to third-rank tensors, when multiplied with $E_{S B}$, and have the same nonzero elements as the tensors $\chi_{s}^{(2)}$ and $\chi_{B}^{(2)}$ [5]. The maximum static electric field $E_{S B}$ at the $S B$ is calculated from the well known equation [7]

$\left|E_{S B}(z=0)\right|=\left(\frac{2 q N_{D} \Phi_{b}}{\varepsilon_{0} \varepsilon_{S}}\right)^{\frac{1}{2}}$,

where $N_{D}$ is the doping density, $\varepsilon_{0}$ is the dielectric permittivity, $\varepsilon_{S}$ is the static dielectric constant, and $\Phi_{b}$ is the band bending at the interface. The latter is given by: $\Phi_{b}=\Phi_{b 0}+V_{b} . \Phi_{b 0}$ is the band bending when no biasvoltage is applied and $V_{b}$ is the value of an applied reversed bias voltage.

The Schottky barrier sample we used for our experiments, is grown on a $n+$ GaAs substrate. The actual barrier is formed by an $0.3 \mu \mathrm{m}$-thick n-type GaAs (doping concentration: $10^{17} \mathrm{~cm}^{-3}$ ) layer and a semitransparant gold film of $80 \AA$ thickness, to allow for laser excitation through the metal top contact. Further details on the sample can be found in [3]. A voltage bias can be applied across the sample between an AuGeNi backsidecontact and the top contact. Capacitance-voltage measurements indicate a value for the Schottky barrier height of $0.90 \mathrm{~V}$.

The pump radiation for the SHG measurements was supplied by a mode-locked titanium sapphire laser that operated at $\lambda=770 \mathrm{~nm}$ and produced trains of $70 \mathrm{fs}$ pulses at a $82 \mathrm{MHz}$ repetition rate. The photon energy at this wavelength is $1.61 \mathrm{eV}$; this is above the band gap of GaAs, which is $1.43 \mathrm{eV}$ at room temperature. The sample was subjected to pump radiation at $45^{\circ}$ angle of incidence. The diameter of the spot was about $100 \mu \mathrm{m}$.

Figure la shows half of the azimuthal anisotropy for two different bias voltages and an average laser power of $4 \mathrm{~mW}$. Figure $1 \mathrm{~b}$ depicts the same, measured with an average laser power of $19 \mathrm{~mW}$. The open circles present data at $0 \mathrm{~V}$ bias, the dots at $-4 \mathrm{~V}$. Figure 1a exhibits a very clear bias dependence of $I_{2 \omega}$, that practically dissappears for $19 \mathrm{~mW}$ excitation, as shown in Fig. $1 \mathrm{~b}$.

The observed $I_{2 \omega}$ can be very well described by

$I_{2 \omega}=\left|A_{s} \exp (\mathrm{i} \varphi)+A_{B} \cos 2 \psi\right|^{2}$.

The measured curves are fitted to (3) with the following three fitting parameters: the interface amplitude $A_{S}$ the phase angle $\varphi$ and the bulk amplitude $A_{B}$. Both the interface and bulk amplitudes are combinations of intrinsic and field induced terms. From the bias field and power

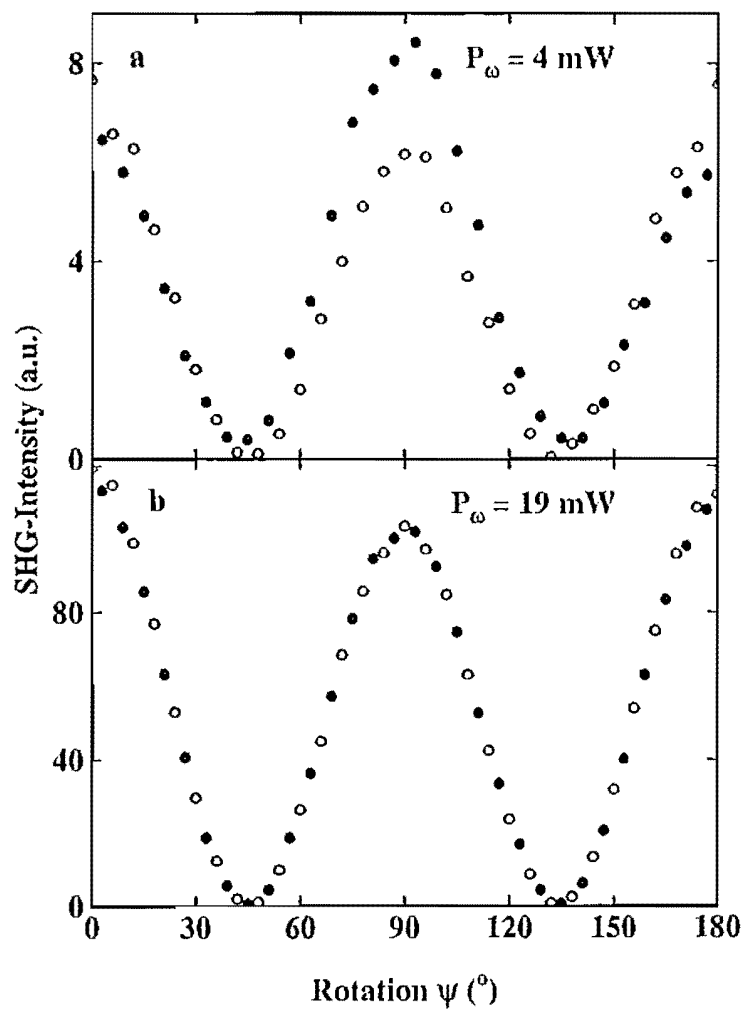

Fig. 1. a Azimuthal anisotropy of the GaAs/Au sample for two different bias voltages, measured with an average laser power of $4 \mathrm{~mW}$ and a pulse width of $100 \mathrm{fs}$. The open circles present data at $0 \mathrm{~V}$ bias, the dots at $-4 \mathrm{~V}$. b The same, measured with an average laser power of $19 \mathrm{~mW}$

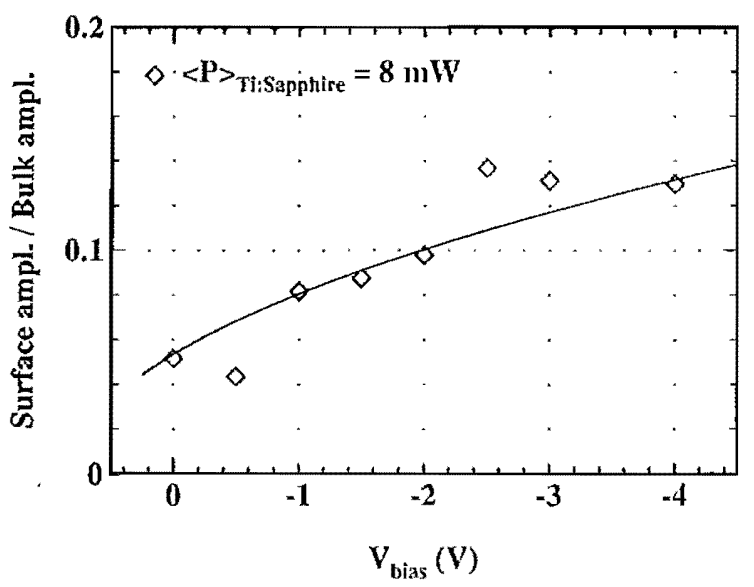

Fig. 2. Bias dependence of the normalised interface contribution measured with a pulse width of $100 \mathrm{fs}$ at an average laser power $P_{\omega}$ of $8 \mathrm{~mW}$. The line is a fit according to formula 4

dependences it followed that the bulk contribution $A_{B}$ is insensitive to changes in the applied voltage and depends linearly on the average pump power, as expected for bulk SHG [1]. We can then normalise the interface amplitude $A_{S}$ to $A_{B}$. From (1 and 2) we expect the normalised interface contribution $A_{S}^{\prime}$ as a function of $V_{b}$ to be of the following form

$A_{S}^{\prime}=C_{0}+C_{1} \sqrt{\Phi_{b 0}-V_{b}}$ 


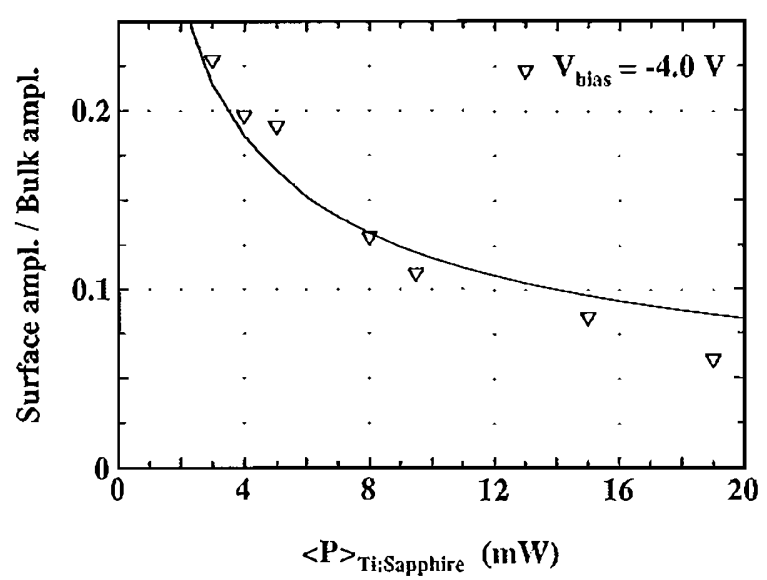

Fig. 3. Power dependence of the normalised interface contribution $A_{S}^{\prime}$ measured at $-4 \mathrm{~V}$ bias with a pulse width of $100 \mathrm{fs}$. The points are connected to guide the eye

Figure 2 exhibits the bias dependence of $A_{S}^{\prime}$ measured with a pulse width of about $100 \mathrm{fs}$ and an average laser power $P_{\omega}$ of $8 \mathrm{~mW}$. The line is a fit, according to (4) with $C_{0}$ and $C_{1}$ as fitting parameters. Using formulas from Sze [7] $\Phi_{b 0}$ is calculated to be equal to $0.8 \mathrm{~V}$. With $C_{0}=0.06$ the fit is in good agreement with our results. $C_{1}$ depends on the average power of the incident laser beam.

Figure 3 displays the power depencence of $A_{S}^{\prime}$ measured at $-4 \mathrm{~V}$ bias and with a pulse width of $100 \mathrm{fs}$. In this figure it can be seen that $A_{\mathrm{s}}^{\prime}$ decreases as the average laser power is increased.

These observations can be interpreted in the following way. The pump laser beam will excite charge carriers since the photon energy is above the band gap of GaAs. Very shortly after excitation a number of holes will reach the top $\mathrm{Au}$ contact, where they neutralize the surface charge, leading to a reduced field strength. In the case that the photo-excited charge density exceeds significantly the intrinsic one, the applied field may be totally quenched by only a small part of the excited holes. A similar effect occurs when the holes are collected in front of the AlGaAs barrier where they screen the applied voltage. It will take some time before the field will be restored. Based on the capacitance per area $(520 \mu \mathrm{mF} /$ $\left.\mathrm{m}^{2}\right)$, the size of the excitation beam $(100 \mu \mathrm{m})$ and the square resistance of the Au film (a few Ohms), we estimated the $\mathrm{RC}$ time constant of this process to be in the order of $10 \mathrm{ps}$. This means that during the laser puls the effective bias is near zero, whereas the system is back to equilibrium before the next puls arrives.

A reduced field strenght results in a lower interface contribution to the $I_{2 \omega}$. The lowering of the interface contribution depends on the time between the generation of the carriers and the generation of the SHG signal. In the experiment one pulse generates the carriers and also generates the SHG signal to probe the field. So the time delay between the pump and the probe is of the order of the pulse width. Repeating these experiments with longer excitation pulses already indicated a time dependence of this effective bias. To probe the time evolution of this field dynamics more directly, puls-probe experiments are momentarily in progress. From the results above we may conclude that SHG in indeed sensitive for the Schottky barrier field and as a result SHG can be used to probe the dynamics of this interface field as well. Similar dynamics studies on a semiconductor surface have recently been reported by Dekorsky et al. [8] using an ultrafast linear electrooptic technique. This effectively probes the depletion layer, and as such, that technique is complementary to our approach.

\section{SHG from magnetic multilayers}

The magnetic properties of thin films and multilayered systems containing ferromagnetic material are a subject of great technological singificance, as these systems are important for magnetic data recording [9]. From a fundamental point of view they provide a fascinating field of research, because of the extraordinary phenomena that are observed. Examples are the change of the magnetization from normal to in plane for thin Fe films [10] and the observed oscillations in the exchange coupling between ferromagnetic films separated by a thin nonmagnetic layer $[11,12]$.

From a technological point of view, better understanding of the magnetic properties of interfaces could lead to improved quality of, e.g., recording heads, while it would be of fundamental interest to determine the role of the interface in the exchange coupling.

Polarized electrons are a sensitive probe for the magnetic properties of clean surfaces [13]. However, their short mean free path makes them difficult to use for studies of buried interfaces. An interface sensitive optical technique would be much more suitable, as it takes advantage of the relatively low absorption of thin metallic films. The widely used Magneto Optical Kerr Effect (MOKE) is a linear technique and with a probing depth of about $50 \mathrm{~nm}$. Though extremely sensitive, it measures the bulk magnetic properties.

On theoretical grounds it has been well established, that magnetic effects should be detectable with SHG $[14,15]$, and first experimental indications were given by Reif et al. [16] and Spierings et al. [17]. The presence of a magnetization does not break the inversion symmetry, as it is described by an axial vector. This means that the basic symmetry argument for interface sensitivity still holds. Possible bulk contributions will only originate from the much smaller quadrupole-type components, that will be neglected in the present analysis. For a more detailed discussion about the fundamental problem of separating bulk and surface SHG, see the paper by Koopmans et al. in [18].

The magnetic properties of the material can be included by introducing a magnetization dependent nonlinear susceptibility tensor: $\chi_{k k}^{(2)}(\mathbf{M})$, as was suggested by Ru-Pin Pan et al. [14]. We will define the interface by the $x, y$-plane, with $x$ in the plane of incidence and parallel to the $x$-component of the wave vector of the incoming light. The magnetization is taken parallel to $y$, and $z$ is parallel to the surface normal pointing into the material. A symmetry analysis shows that we can distinguish ten- 


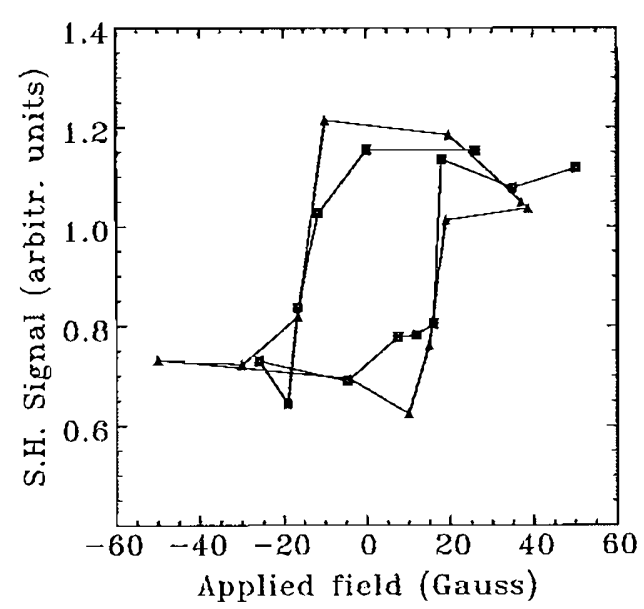

Fig. 4. MSHG hysteresis for a $\mathrm{CO} / \mathrm{Au}$ interface

sor elements that are even and odd, respectively, in the magnetization [14] thus we may write

$E(2 \omega)=\left[\chi_{\text {even }}^{(2)}(\mathbf{M})+\chi_{\text {odd }}^{(2)}(\mathbf{M})\right] E^{2}(\omega)$,

where $\chi_{\text {even }}^{(2)}(\mathbf{M})$ and $\chi_{\text {odd }}^{(2)}(\mathbf{M})$ are linear combinations of allowed tensor elements, and $E(\omega)$ is the fundamental field at the interface. Changing the sign of $\mathbf{M}$ causes a phase change of $180^{\circ}$ between the two contributions in (5), and leads to a different SH intensity. We now define the relative magnetic effect for Magnetizationinduced SHG (MSHG) as

$\varrho=\frac{I\left(2 \omega, \mathbf{M}^{+}\right)-I\left(2 \omega, \mathbf{M}^{-}\right)}{I\left(2 \omega, \mathbf{M}^{+}\right)+I\left(2 \omega, \mathbf{M}^{-}\right)}$

where $I\left(2 \omega, \mathbf{M}^{+}\right)$and $I\left(2 \omega, \mathbf{M}^{-}\right)$are the SH intensities for magnetic saturation parallel and antiparallel, respectively, to the $y$-axis.

Figure 4 shows the observed MSHG signal from a $\mathrm{Co} / \mathrm{Au}$ sample $(50 \mathrm{~nm} \mathrm{Co}+5 \mathrm{~nm} \mathrm{Au}$ on a quartz substrate), showing a clear magnetic effect. For the experiment we used the frequency doubled output at $532 \mathrm{~nm}$ of a $Q$-switched Nd-YAG laser, incident at an angle of 45 degrees on the sample that was mounted between the poles of an electromagnet. We used the $p, p$ configuration, with the magnetization perpendicular to the plane of incidence and in the plane of the sample. The result not only shows the presence of an effect, but more importantly, that this is not depending on the applied field, as the presence of the hysteresis loop clearly indicates. To prove that the interfaces and not the bulk, are the sources for MSHG, we have varied the number of interfaces. The observed variation of the magnetization induced effects are, indeed, consistent with our model that only takes interface contributions into account [19]. Moreover, the signals did not depend on the bulk amount of Co in the various samples. A detailed description of our quantitavive analysis goes beyond the scope of this paper and will be published elsewhere [20]. The general approach is that we calculate the fundamental fields at the interfaces from multiple reffection theory [21]. Using the relevant tensor elements and the boundary conditions for a non- linear source polarization at an interface, we derive the discontinuity of the $\mathrm{SH}$ field at the interface [1]. The total SH generated by the sample is calculated by using again multiple reflection theory (now for $2 \omega$ ), and summing over all interfaces.

From Fig. 4 it follows that the magnetic contribution is about $10 \%$. This is a huge effect, but in good qualitative agreement with recent calculations from Hubner for other materials, based on spin-dependent band structures. From his calcualtions it also followed that for $\mathrm{Ni}$ at $532 \mathrm{~nm}$, the nonlinear magnetic susceptibility is near zero, in accordance with recent experiments we performed in our laboratory (unpublished results).

A more direct way to prove the intrinsic surface/interface sensitivity is to measure the MSHG response during the growth of a magnetic thin film. In collaboration with Prof. J. Kirschner's group (MPI, Halle), we recently started an insitu MSHG investigation of epitaxially grown Co films on a $\mathrm{Cu}(001)$ substrate. For the $\mathrm{SHG}$ experiments we used the $800 \mathrm{~nm}$ output of a Ti : Sapphire (Tsunami) laser operating at a repetition rate of $82 \mathrm{MHz}$ and a pulse width of about $100 \mathrm{fs}$. The incoming laser light was filtered and focused onto the sample, leading to a pulse intensity of about $16 \mu \mathrm{Jcm}^{-2}$. At an angle of incidence of $35^{\circ}$, we have studied the pp polarization combination (i.e., both fundamental and SH are polarized in the plane of incidence). No analyzer was needed, because the $s$-polarized SH output was negligible, as predicted by theory. Appropriate filtering was used before the signal was detected by a photomultiplier in combination with a lock-in amplifier. The MOKE hysteresis were taken in the longitudinal configuration at an angle of incidence of $45^{\circ}$, with the external field applied along the easy axis $y$.

The samples were prepared in an UHV system with a base pressure of $7 \times 10^{-11}$ mbar. Further details on sample preparation can be found in [23].

Figure 5 shows the relative MSHG signal and the amplitude of the MOKE hysteresis $\left(M_{r}\right)$ as a function of

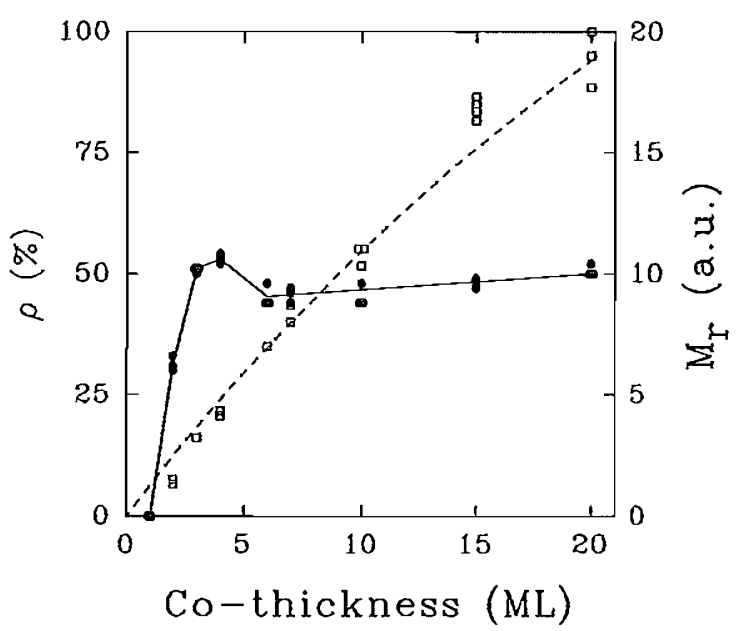

Fig. 5. Thickness dependence of the amplitude of the MOKE hysteresis $\left(M_{r}\right)$ and the relative magnetic effect in pp MSHG for Co on $\mathrm{Cu}(100)$; squares/long dashed line: MOKE data and fit; filled circles: $\varrho(p p)$; solid line: $\varrho(p p)$ and guide to the eye 
the Co-film thickness. The MOKE amplitude is showing an almost linear increase with increasing thickness. The small devitation from linearity is due to the non negligible absorption, which is taken into account in the standard Lambert-Beer's type of analysis. The MOKE data are accurately described by bulk refractive indices for Co thicknesses above $3 \mathrm{ML}$.

The thickness dependence of the pp MSHG is completely different. Figure 5 shows that $\varrho(p p)$ changes only up to $6 \mathrm{ML}$, whereafter it becomes nearly constant and varies only a few percent for Co layers ranging from 6 to 20ML. Due to the fact that we excite with a focused beam, surface inhomogeneities of the substrate cause poor reproducebility of the absolute signals. Within the (large) experimental error the SH signal was nearly constant. However, the value of $\varrho(p p)$, as it represents a relative effect, reproduced within a few \%. A second method to determine the origin of the MSH is changing the Co/vacuum interface by $\mathrm{CO}$ absorption. We observed that the signals changed until a dosage of 1 Langmuir (1 $\mathrm{L}=10^{-6}$ torr.s), whereafter they became constant and remained unchanged until at least $40 \mathrm{~L}$. The original value of $\varrho \sim 0.45$ increased to $\varrho \sim 0.70$. Comparable effects have been observed on absorbing $\mathrm{O}_{2}$ and for different $\mathrm{Co}$ film thicknesses. The fact that $\varrho(p p)$ is nearly constant for Co films between 6 and $20 \mathrm{ML}$, and the sensitivity to gas absorption clearly proof interface sensitivity of MSHG.

Gas adsorption usually strongly reduces the $\mathrm{SH}$ generated by metal surfaces [2]. We have observed that a dosage of a few Langmuirs of $\mathrm{O}_{2}$ to magnetized $\mathrm{Ni}(110)$ and $\mathrm{Fe}(110)$ crystals reduces the pp SH signal, generated from a $532 \mathrm{~nm} \mathrm{Nd:YAG} \mathrm{beam,} \mathrm{by} \mathrm{up} \mathrm{to} \mathrm{a}$ factor of 20 depending on initial cleanliness. We therefore conclude that the $\mathrm{CO}$ eliminates all SH contribution by the $\mathrm{Co} /$ vacuum-interface, and not only the elements that are odd in $\mathbf{M}$, as was suggested by Reif et al. [16]. This we still use for our quantitative analysis of the results for pp.

Multiple-reflection theory demonstrates that at both interfaces the ratio $E_{z}(\omega) / E_{x}(\omega)$, is virtually independent of the thickness of the Co film, and we conclude that we need not distinguish between $X_{j x x}$ and $X_{j z z}$. Furthermore time-inversion symmetry suggests a phase difference of $90^{\circ}$ between odd and even tensor elements [14], this value was also found in our experiments on $\mathrm{Co} / \mathrm{Au}$ multilayers [19]. So $\chi_{z x x}, \chi_{x x z}, i \chi_{x x x}$ and $i \chi_{z x z}$, with $\chi_{j k 1}$ all real, fully describe the interfaces.

Using the bulk refractive indices from [24] and an interface refractive index $\varepsilon^{\prime}(\omega)=\varepsilon^{\prime}(2 \omega)=1$ [25], we obtain an equally good description of the results with any combination of one odd and one even tensor element. Although a detailed study of all polarization combinations will be necessary to resolve the values of all the individual tensor elements, their precise value is not of vital importance to come to a general description of the system. From our analysis we find similar strengths and opposite phases of the tensor elements at both interfaces. The latter is intuitively expected from the consideration that we have an alternation of magnetic and nonmagnetic materials.

The results of the MSHG experiments in the range of
1-6 ML are, of course, not explained by simple multiplereflection arguments. We observe quite drastic changes for $s p$, and for $p p$ we measure an overshoot-like structure. Although these effects might be caused by strain induced changes of $\chi$, one could speculate on a different origin, namely the electronic coupling of the Co/vacuum and the $\mathrm{Co} / \mathrm{Cu}$-interface. The $\mathrm{Co} / \mathrm{Cu}$-system is well known for the appearance of quantum-well states [26]. Because of the spin-orbit coupling the spin up and spin down electrons will be affected differently, and the oscillations should be reflected in the SH intensities. This exciting area will be a subject of future studies.

We may conclude that the magnetization induced second-harmonic generation does appear to be sensitive to magnetic interfaces, as was shown on Co/Au and $\mathrm{Co} / \mathrm{Cu}$ systems. The observed magnetic effects are huge and open a new field exploring the (magnetic) structure of these interesting and important interfaces. Combining these results with pump-probe measurements can lead to the study of the spin dynamics. Other future possibilities may be the imaging of interface domain structure.

As a general conclusion, we can state that SHG can be a unique tool, espescially to study the properties of buried interfaces. It has been shown that this even works for non centro symmetric structures like GaAs. Electric and magnetic field induced effects can lead to new ways to study the electronic and magnetic properties of interfaces. The possibility to combine this with time-resolved measurements is especially attractive.

Acknowledgements. The work described here is the result of the ongoing efforts of many people in our group in Nijmegen. In particular, I want to mention H.A. Wierenga, W. de Jong, M.W.J. Prins, A.F. van Etteger, and C.A. van' 't Hof. In addition I would like to mention the very fruitful collaboration with $R$. Vollmer, A. Kirilyuk, H. Schwabe and J. Kirschner from Halle and with P.J. van Hall, Eindhoven. This work was part of the research program of the Stichting voor Fundamenteel Onderzoek der Materie (FOM) and financially supported by the Nederlandse organisatie voor Wetenschappelijk Onderzoek (NWO).

\section{References}

1. Y.R. Shen: The Principles of Nonlinear Optics (Wiley, New York 1984)

2. G.L. Richmond, J.M. Robinson, V.L. Shannon: Prog. Surf. Sci. 28, 1 (1988)

3. P.C.M. Christianen: PhD. thesis, University of Nijmegen (1993)

4. P.C.M. Christianen, P.J. van Hall, H.J.A. Bluyssen, J.H. Wolter: Semicond. Sci. Technol. 9, 707 (1994)

5. J. Qi, M.S. Yeganeh, I. Koltover, A.G. Yodh, W.M. Theis: Phys. Rev. Lett. 71, 633 (1993)

6. T. Stehlin, M. Feller, P. Guyot-Sionnest, Y.R. Shen: Opt, Lett. 13, 389 (1988)

7.S.M. Sze: Physics of Semiconductor Devices, 2nd edn. (Wiley, New York 1981)

8. T. Dekorsky, T. Pfeifer, W. Kütt, H. Kurz: Phys. Rev B 47, 3842 (1993)

9. L.M. Falicov et al.: J. Mater. Res 5, 1299 (1990)

10. A. Vaterlaus, M. Stampanoni, M. Aeschlimann, F. Meier: J. Appl. Phys. 64, 5331 (1988)

11. P. Grünberg, R. Schreiber, Y. Pang, MB. Brodsky, H. Sowers: Phys. Rev. Lett. 57, 2442 (1986)

12. A. Cebollada, R. Miranda, C.M. Schneider, P. Schuster, J. Kirschner: J. Magn. Magn. Mater. 102, 25 (1991) 
13. See for example: Polarized Electrons in Surface Physics, ed. by R. Feder (World Scientific, Singapore 1985)

14. Ru-Pin Pan, H.D. Wei, Y.R. Shen: Phys. Rev. B 39, 1229 (1989)

15. W. Hübner, K.H. Bennemann: Phys. Rev. B 40, 5973 (1989)

16. J. Reif, J.C. Zink, C.M. Schneider, J. Kirschner: Phys. Rev. Lett. 67, 2878 (1991)

17. G. Spierings, V. Koutsos, H.A. Wierenga, M.W.J. Prins, D. Abraham, Th. Rasing: Surf. Sci. (IV-12, ICSS-8, The Hague, 1992) 287, 747 (1993); J. Magn. Magn. Mater. (Symposium Magn. Thin Films, Lyon, 1992) 121, 109 (1993)

18. B. Koopmans et al.: Appl. Phys. A (in press)

19. H.A. Wierenga, M.W.J. Prins, D.L. Abraham, Th. Rasing: Phys. Rev. B 50, 1282 (1994)
20. H.A. Wierenga, M.W.J. Prins, Th. Rasing: Physica B (in press)

21. Handbook of Optical Constants of Solids, ed. by E.D. Palik (Academic, Orlando 1985)

22. R. Gamble, P.H. Lissberger: J. Opt. Soc. Am. A 5, 1533 (1988)

23. H.A. Wierenga, W. de Jong, M.W.J. Prins, Th. Rasing, R. Vollmer, A. Kirilyuk, H. Schwabe, J. Kirschner; Phys. Rev. Lett. (submitted)

24. P.B. Johnson, R.W. Christy: Phys. Rev. B 9, 5056 (1974); Phys. Rev. B 6, 4370 (1972)

25. P. Guyost-Sionnest, W. Chen, Y.R. Shen: Phys. Rev. B 33, 8254 (1986)

26. J.E. Ortega, F.J. Himpsel, G.J. Mankey, R.F. Willis: Phys. Rev. B 47, 1540 (1993) 